\title{
Macronutrient balances and obesity: the role of diet and physical activity
}

\author{
Arne Astrup* \\ Research Department of Human Nutrition, KVL, Rolighedsvej 30, DK-1958 Frederiksberg C, Denmark
}

Accepted: 7 May 1999

\begin{abstract}
Observational cross-sectional and longitudinal studies suggest that a high fat diet and physical inactivity are independent risk factors for weight gain and obesity. Mechanistic and intervention studies support that fat possesses a lower satiating power than carbohydrate and protein, and a diet low in fat therefore decreases energy intake. The effect of dietary fat on energy balance is enhanced in susceptible subjects, particularly in sedentary individuals with a genetic predisposition to obesity who consume a high fat diet.

Dietary carbohydrate promotes its own oxidation by an insulin-mediated stimulation of glucose oxidation. In contrast, high fat meals do not increase fat oxidation acutely. A sedentary life-style and low physical fitness cause a low muscular fat oxidation capacity, and the consumption of a high fat diet by these individuals promotes fat storage in a synergistic fashion.

Ad libitum low fat diets cause weight loss proportional to pre-treatment body weight in a dose-dependent way, i.e. weight loss is correlated positively to the reduction in dietary fat content. Increased physical activity prevents relapse after weight loss and studies have shown that those who keep up a higher level of physical activity are more successful in maintaining the reduced body weight. In conclusion, important interactions exist between genetic make up, dietary fat and physical fitness, so that a low fitness level and susceptible genes reduce muscular fat oxidation capacity which may decrease the tolerance of dietary fat. Increasing daily physical activity and reducing dietary fat content may be more effective when combined than when separate in preventing weight gain and obesity.
\end{abstract}

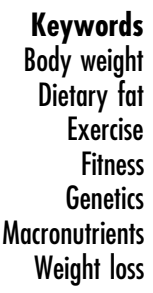

\section{Key messages}

- An energy dense, high fat diet and low levels of daily physical activity are independent risk factors of weight gain and obesity in genetically predisposed individuals.

- Important interactions may exist between genetic make up, dietary fat and physical fitness, so that a low fitness level reduces muscular fat oxidation capacity, which may decrease the tolerance of dietary fat.

- Increasing daily physical activity and reducing dietary fat content may be more effective in combination than separately in preventing weight gain and obesity ${ }^{1}$.

\section{Introduction}

The rapid increase in the global prevalence of obesity has been characterized as an epidemic by the $\mathrm{WHO}^{2}$. In most affluent populations the prevalence of obesity (BMI $>30 \mathrm{~kg} \mathrm{~m}^{-2}$ ) among adults is $10-40 \%$. In the USA obesity affects $25 \%$ of the adult population and its major complications, e.g. diabetes, ischaemic heart disease, hypertension, stroke, certain cancers and disability, may account for $5-10 \%$ of all health costs.

The abundant food energy supply in the majority of populations has removed limited energy availability as a restricting factor for the development of obesity. The proliferation of cheap, highly palatable, high fat, instant, fast and pre-packaged foods is assumed to contribute to overconsumption and to divest consumers of control over preparation of their own foods (Fig. 1). However, obesity is generally accepted to develop on the background of a genetic predisposition, which comes to expression when susceptible individuals are exposed to a life style characterized by physical inactivity and an abundant availability of energy dense, high fat and palatable foods, and inappropriate meal patterns ${ }^{2-4}$.

The energy density of foods has been shown to play a major role in the regulation of the spontaneous energy intake, but macronutrient specific properties may also be important in the propensity to major weight gain and 


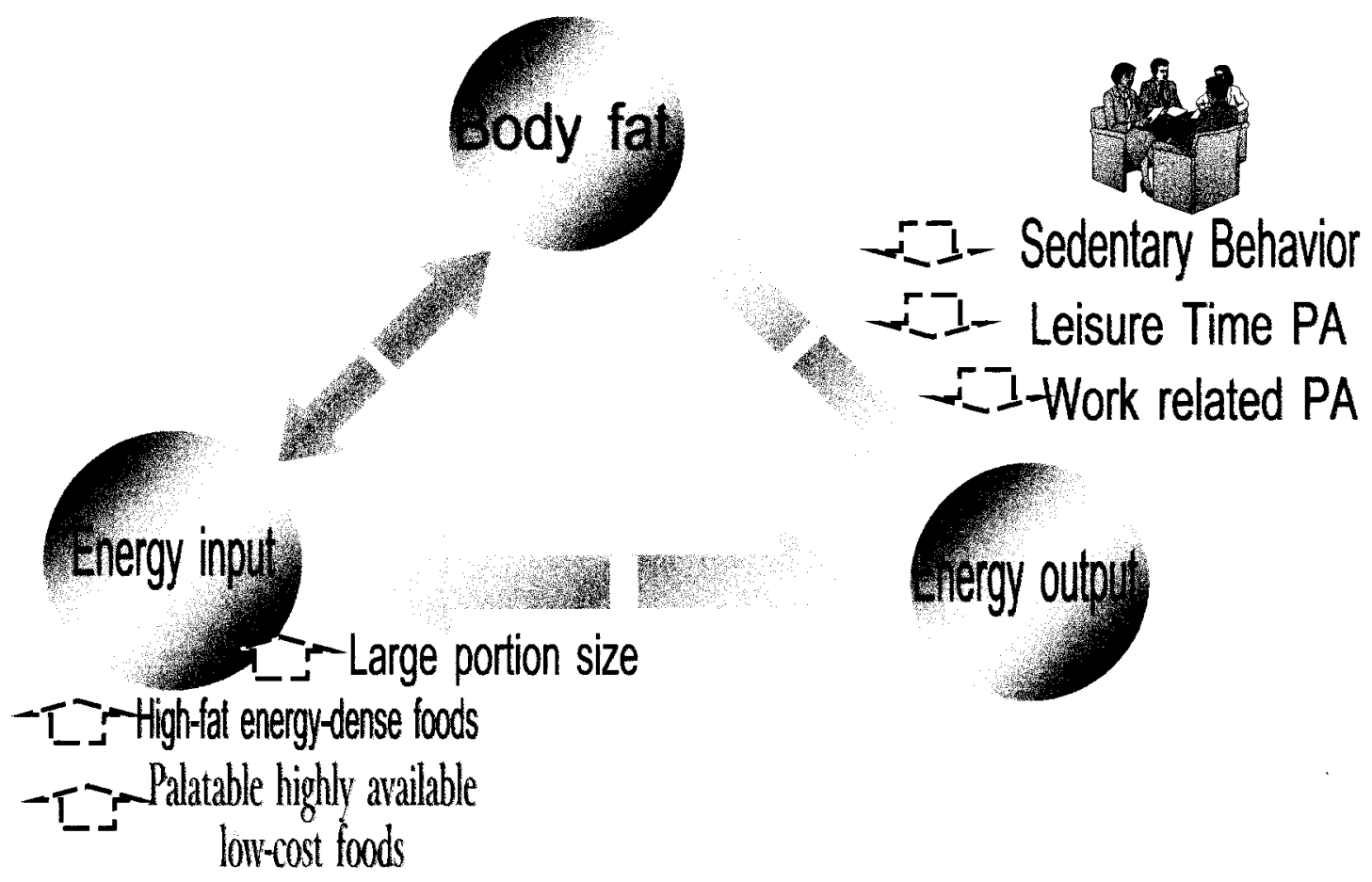

Fig.1 A schematic overview of the most important factors causing passive overconsumption (increased energy input) and decreased energy output, factors which, particularly in genetically predisposed individuals, promote weight gain and obesity. The low physical activity level may promote a positive fat balance by: 1) A low energy output; 2) A low fitness with accompanying low fat oxidation capacity; and 3) An impaired appetite control

obesity. The propensity to gain weight on an energy dense, high fat diet may be highly dependent on the daily level and pattern of physical activity, and on the presence of obesity susceptible genes (Fig. 1).

\section{The principle of macronutrient balance}

The traditional concept of the energy balance equation, which describes weight gain as an excessive positive energy imbalance, can be replaced usefully by a series of macronutrient balance equations in which gains in body fat stores are viewed specifically as an imbalance of fat ${ }^{5}$. The background for this replacement is that calories are not equal in their metabolic effects, that interconversion between the 4 macronutrients is negligible and that an oxidative hierarchy operates in inverse proportion to the size of available stores for each macronutrient. Amino acid, glucose and alcohol oxidation adjust readily to protein, carbohydrate, and alcohol intakes. Seen over a period of a few days, regulation appears to be geared primarily to suppress hunger through gastrointestinal signals, and to maintenance of appropriate glycogen reserves, and to a lesser extent to the maintenance of body fat stores.

Alcohol is most readily oxidized because it cannot be stored. Oxidation of carbohydrate and protein are also under tight auto-regulatory feedback control: oxidation increases in direct response to intake. In contrast, there is virtually no acute feedback between fat intake and fat oxidation. Fat oxidation is primarily a function of the gap between total energy expenditure and the amounts of alcohol, protein and carbohydrate energy consumed $^{6}$, resulting in a much less accurately maintained fat balance. Although regulatory responses serving to achieve fat balance exist, their effectiveness in inhibiting the expansion of the fat mass seems to be limited. Leptin, a hormone secreted from adipose tissue at an increased rate when the fat mass is expanded inhibits food intake and increases sympathetic nervous system activity through a central action ${ }^{7}$. Mutations in the leptin gene and the leptin receptor gene have been found to result in severe obesity. However, these are extremely rare exceptions and the finding that obese subjects remain obese in spite of 10-fold higher circulating leptin concentrations suggests that these lipostatic mechanisms are insufficient to restrict energy intake in this category of individuals. However, defects in other lipostatic mechanisms may play a role in simple obesity. Dietary fat oxidation relates poorly with daily variations in fat consumption. This is a probable explanation as to why obesity develops particularly among individuals with a genetically determined low fat oxidation capacity when the diet is high in fat and when physical activity is limited ${ }^{8}$. Thus, the enlarged body fat mass of obesity may represent an adaptation to a high-fat diet in subjects with a low fat oxidation capacity caused by genetic make up and a low physical fitness. Long-term fat and energy balances tend to 
remain close to zero over prolonged periods once a weight maintenance plateau has been reached (i.e. less than $2-3 \%$ error relative to energy turnover considered over one year).

\section{Diet composition, appetite regulation and obesity}

To establish a causal link between exposure to a diet component and weight gain and obesity should be based on all available lines of evidence, i.e. feeding studies in experimental animals, observational crosssectional and longitudinal human studies, short-term experimental and mechanistic studies and intervention studies. These lines of evidence will be reviewed briefly.

\section{Experimental studies on diet composition and energy intake}

Considering the problem of weight gain and obesity, the issue is to determine the nature of the dietary components that facilitate consumption and lead to a positive energy balance. Dietary components can influence energy intake by acting during consumption and in the period following eating (post-ingestive period). Dietary protein exerts a potent effect on satiety and provides the strongest inhibition of energy intake. Delivered in iso-energetic amounts, high protein meals generate stronger satiety than high carbohydrate meals9. Carbohydrates also contribute markedly to the satiating efficiency of foods and exert clear effects on satiety. This evidence forms part of the 'glucostatic' or 'energo-static' mechanism of appetite control. A variety of carbohydrates including glucose, fructose, sucrose and maltodextrins exert fairly similar effects on satiety, when given in a pre-load they suppress later intake by an amount roughly equivalent to their energetic value. The time course of the suppressive action may vary according to the rate at which the high carbohydrate loads are metabolized, as demonstrated by the amylose content of starch. There is also some evidence that the degree of satiety is related to the glycaemic index of the carbohydrate in the food. Resistant starch (dietary fibre) may prolong satiety by modulating the time course of the pre- and postabsorptive satiety signals. Fibre can also limit intake by lowering the energy density of the food, thus allowing time for satiation (and early satiety signals) to occur before large amounts of energy have been consumed.

An active area of interest involves comparisons of fat and carbohydrate. Subjects exposed to high fat foods for weeks tend to overconsume energy. This effect depends in a large part on the high energy density of the high fat foods, and the overeating effect has been referred to as passive overconsumption ${ }^{4,9-11}$. It should be noted that the stimulatory effect of fatty foods on energy intake is not only caused by their high energy density but also by the probable facilitatory action of fat in the mouth ${ }^{12}$. It has been known for many years that offering subjects high fat or high carbohydrate foods which have been manipulated to be equally energy dense eliminates the high fat overeating phenomenon in normal non-genetically predisposed individuals ${ }^{13}$.

The passive overconsumption effect of fat on energy intake is the result of an action during consumption (it is an intra-meal effect). The large amounts of fat energy consumed do not appear to generate equivalent effects on post-ingestive satiety. Therefore fat has a proportionally weaker effect on satiety relative to the amount of energy consumed. In studies where energetically equivalent preloads of fat and carbohydrate have been delivered, it appears that fat does exert a weaker satiating effect (on a Joule for Joule basis) than the other macronutrients.

A large body of short-term studies on appetite and energy intake unequivocally show that fat is less satiating than carbohydrate and protein when compared Joule for Joule, and that high fat foods are more likely to induce passive overconsumption and weight gain than low fat foods ${ }^{10-14}$.

\section{Observational studies on diet composition and obesity}

Numerous cross-sectional studies have clearly demonstrated positive associations between the proportion of total energy intake covered by fat and body fatness, and inverse associations between carbohydrate and body fatness ${ }^{14,15}$. In contrast, a number of longitudinal studies have been unable to establish any association between self-reported dietary fat and carbohydrate intakes and subsequent weight change ${ }^{16}$. However, evidence based on observational studies looking for associations between habitual dietary macronutrient intakes and body fatness has a number of limitations because of the reliance on information about dietary intakes given by the subjects under examination. Valid information on dietary fat intake is difficult to achieve in populations that are recommended to reduce fat intake, because they may eat a more healthy diet during the dietary survey or they may under-report fat intake. Further, day-to-day fluctuations in dietary fat intake, which are not captured by reporting average fat intake, could lead to episodic bouts of fat storage, as fat oxidation is not increased in response to acute increases in fat intake. It is well established that overweight and obese subjects under-report their energy intake by $30-40 \%{ }^{17}$, and fat may be overrepresented in this underreporting ${ }^{18}$. Studies in health conscious populations show that high fat foods are underreported, whereas low fat foods are overreported $^{19}$, and it seems to be easier to 
demonstrate associations between dietary fat intake and subsequent weight changes in less health conscious populations, such as in $\mathrm{China}^{20}$, than in EU and United States ${ }^{16}$.

In conclusion, cross-sectional studies quite consistently support that a high fat, low-carbohydrate diet contributes to maintain the obese condition, but because of the methodological limitations in obtaining valid information about dietary macronutrient intakes in diet conscious and overweight populations the consistent outcome should not be given major weight in the assessment of the dietary fat and body fat relationship.

\section{Intervention studies}

Randomized controlled ad libitum low fat, high carbohydrate intervention studies show mean weight loss ranging between 0 and $10 \mathrm{~kg}$ in the intervention group, as compared to a control group maintaining their usual diet or consuming a medium to high fat $\operatorname{diet}^{14}$. This large variability and inconsistent outcome has produced doubt about the effectiveness of a low fat diet in the prevention and treatment of overweight and obesity, and questioned the role of dietary fat in body fatness. Trials assessing the effect of ad libitum low fat diets on body weight may suffer from uncertainties about adherence to the diet composition. Reviews taking factors such as pretreatment body weight, reduction in dietary fat, and compliance into consideration have produced more coherent views ${ }^{14}$. A recent systematic review concluded that dietary fat does play an important role in human obesity ${ }^{21}$. Based on 28 intervention trials Bray and Popkin found that a reduction of $10 \%$ in the proportion of energy from fat was associated with a reduction in weight of $16 \mathrm{~g} / \mathrm{d}$, which corresponds to a weight loss of $2.9 \mathrm{~kg}$ over 6 months ${ }^{21}$. Our recently conducted meta-analysis of the results from 18 controlled, ad libitum, low fat, 2-12 months trials on weight change involving 1728 normal and overweight individuals of both genders shows that a reduction in dietary energy from fat by $12 \%$ is associated with a spontaneous weight loss of $2.5 \mathrm{~kg}$ (95\% CI, 1.5-3.5; $P<0.0001)$ more in the intervention than in the control group ${ }^{22}$. The analysis indicated that only minor weight loss occurred in groups with body weights in the normal range $(60-72 \mathrm{~kg})$, but this group did not experience the slight weight gain seen in the control groups consuming normal fat diets. Weight loss increased progressively with increasing body weight. If extrapolated to a body weight of $88 \mathrm{~kg}$ (corresponding to a BMI $\sim 30 \mathrm{~kg} / \mathrm{m}^{2}$ ), and assuming a $10 \%$-point fat reduction, the predicted weight loss would be $4.4 \mathrm{~kg}$ (95\% CI, 2.0 to $6.8 \mathrm{~kg}$ ). These analyses show that a reduction in dietary fat without restriction of total energy intake causes weight loss in a dose-dependent fashion and may produce a modest, but clinically relevant, weight loss in overweight subjects. Therefore, statements such as 'Diets high in fat do not appear to be the primary cause of the prevalence of excess body fat in our society, and reductions in [dietary] fat will not be the solution' ${ }^{23}$ and in the longer term, fat consumption within the range of $18-40 \%$ appears to have little if any effect on body fatness ${ }^{24}$ are unsubstantiated.

\section{The genetic influence of dietary fat susceptibility}

Whereas low fat diets may be effective in preventing weight gain, their efficacy in causing weight normalization in obese subjects may be much less pronounced. Rats fed a high fat diet have been shown not to return to their normal weight when switched back to a low fat diet ${ }^{25}$. This observation was extended by the finding that animals switched to a low fat diet after having been fed a high fat diet for a short time ( 4 months) reduced their weight to normal levels, but animals fed the high fat diet for 7 months failed to reduce body weight to control values ${ }^{26}$. The susceptibility to gain weight on high fat diets is highly genetically determined in rodents. Recently, it has been shown that the Mahogany gene seems to determine the susceptibility to become obese on a high fat diet in mice ${ }^{27}$. It is likely that the same phenomenon exists in humans, but the role of the Mahogany gene remains to be elucidated.

Longitudinal studies have suggested that the development of obesity is promoted by a high fat diet, particularly in subjects with a familial predisposition ${ }^{28}$, which is probably genetically determined. A low ability to oxidize fat has been proposed as a genetically determined trait, and it may be expressed phenotypically as a positive fat balance when consuming a high fat diet, which may cause weight gain and obesity ${ }^{8}$. Why does dietary fat only promote passive overconsumption and weight gain in certain susceptible individuals? This question remains to be answered, but ongoing research finds it difficult to dissociate fat's high energy density from biochemical properties related to its metabolism. Altered mechanisms involved in partitioning and oxidation of fat may be important. Fat oxidation rate is determined by the individuals energy requirement, energy balance, dietary fat content, physical fitness, plasma insulin, oestrogen levels and sensitivity, circulating lipid substrates, skeletal muscle uptake and activity of oxidative enzymes. After controlling for dietary fat intake, energy balance and aerobic capacity, fat oxidation rate has been shown to be a family trait with a heritability of $30 \%{ }^{29}$. A low fat oxidation for a given dietary fat content is a risk factor for subsequent weight gain, and is prevalent among formerly obese subjects with a family history of obesity 

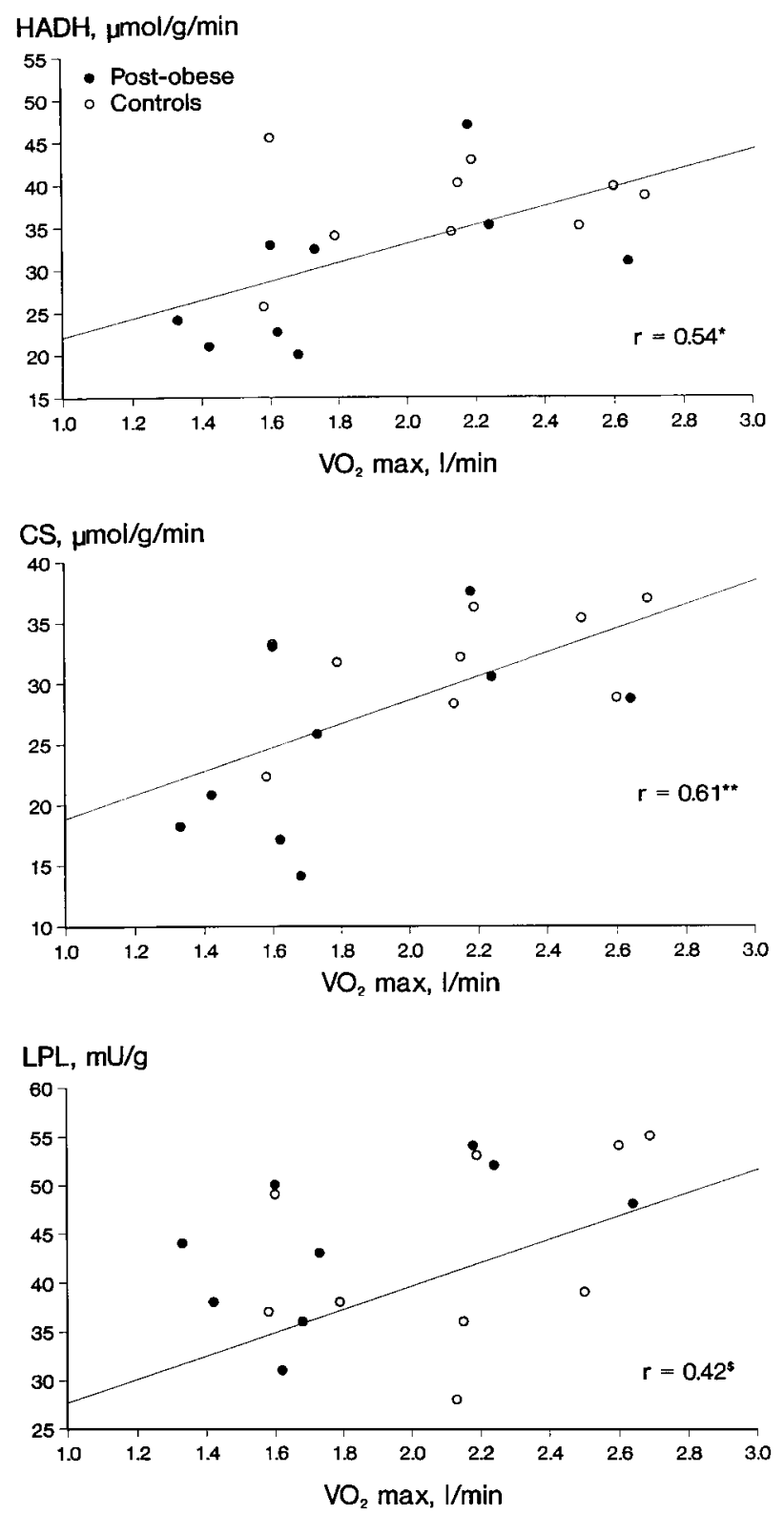

Fig. 2 Formerly obese subjects with the predisposition to obesity have an impaired whole body fat oxidation rate. The present study found that the lower fat oxidation of the formerly obese could be attributed to lower activities of important fat oxidation enzymes in skeletal muscle as compared with the controls. (HADH, $\beta$-hydroxyacyl-CoA-dehydrogenase; CS, citrate synthase; LPL, lipoprotein lipase). However, when $\mathrm{V}_{02 \max }$ was taken into consideration, the lower aerobic capacity of the formerly obese was almost entirely explained by the difference in enzyme activities. It remains to be determined whether these differences are due to differences in physical activity or are genetically determined. (Reproduced with permission from reference 31 )

(Fig. 2). Strictly controlled metabolic studies have demonstrated that the impaired oxidative capacity of obese and post-obese subjects limits fat oxidation, particularly post-prandially and during exercise. Fat mobilization through lipolysis is intact but skeletal muscle uptake and oxidation is restricted ${ }^{30}$ and possible responsible mechanisms are altered responsiveness of thyroid hormones and sympathetic nervous system activity to a high fat diet, high insulin sensitivity, low LPL-activity, and $\beta$-hydroxy-acyl-CoA-dehydrogenase in the muscle fibres ${ }^{31}$. The mechanisms linking the low fat oxidation and positive fat balance to the stimulation of appetite and energy intake are poorly understood.

\section{Interaction between dietary fat and physical activity}

The prevalence of obesity has, in a number of countries, continued to rise despite a reported reduction in the proportion of calories from fat in the national diets. Having been observed in the USA and the UK, the phenomenon has been named "The American Paradox", 32 . Among the explanations suggested for the apparent contradiction have been a concomitant over-riding effect of decreasing physical activity, overconsumption of highly palatable energy dense, carbohydrate-rich, low fat products ${ }^{3}$, underreporting of fat consumed in dietary surveys, and obesity being an infectious disease caused by an adenovirus $^{33}$. It should, however, be stressed that a high dietary fat content has never been claimed to be the only environmental factor responsible for obesity. A sedentary lifestyle with a low level of energy expended on physical activities is another causative factor, which interacts with dietary fat content. This is emphasized in the studies by Stubbs et al. ${ }^{10,11}$, which are summarized in Fig. 3. They demonstrate that even in non-obese individuals, dietary fat tolerance is greatly lowered

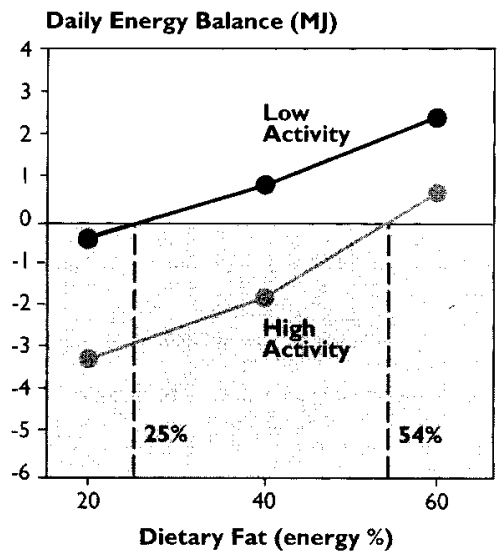

Fig. 3 The interaction between energy balance, level of physical activity and dietary fat content in non-obese individuals. The relations are established from studies in which the energy balance was studied by measurement of daily energy intake and energy expenditure, either in respiratory chambers (low activity) or freeliving by doubly labelled water (high activity), during 3 different diet compositions $^{10,11}$. It appears that with the low physical activity level negative energy balance was achieved on a diet containing not more than $25 \%$ fat-energy, whereas the high activity level allowed the consumption of a diet with fat contents up to $54 \%$ fat-energy before a positive energy balance was reached 
by a sedentary life style. The decrease in physical activity observed in population studies may, therefore, be responsible for the continuing increase in the prevalence of obesity. Another important issue is the validity of the dietary surveys reporting the decrease in fat consumption in, for example, the American population. Whereas the surveys' estimates of dietary fat energy- $\%$ show a decrease from $42 \%$ in 1965 to $37 \%$ in 1987, per capita values of fat intake and dietary fat energy- $\%$ based on food availability have increased from 42 to 43 energy- $\%$ in the same period ${ }^{14}$. Although the food disappearance data do not include some types of food losses and waste (e.g., trimming fat from meats), and commodities used in pet foods are not subtracted from the total amounts available in the food supply, the increasing lack of agreement is strongly suggestive of a substantial under-reporting of dietary fat in the surveys. This is not surprising as one would expect subjects to report more healthy eating habits when asked to give information to nutrition experts. Moreover, the well described under-reporting of energy and fat consumption among obese subjects may have increasing weight in surveys conducted in a population where the majority are either overweight or obese.

Therefore, the increasing prevalence of obesity may be caused by the decreasing level of physical activity and the very modest decrease in dietary fat intake, if any, is not sufficient to retard this development.

\section{Conclusion}

An energy dense, high fat diet and low levels of daily physical activity are independent risk factors of weight gain and obesity in genetically predisposed individuals. Important interactions may exist between genetic make up, dietary fat and physical fitness, so that a low fitness level reduces muscular fat oxidation capacity, which may decrease the tolerance of dietary fat. Moreover, susceptible genes may increase the vulnerability to these two lifestyle factors. Increasing daily physical activity and reducing dietary fat content may be more effective in combination than separately in preventing weight gain and obesity ${ }^{1}$.

\section{References}

1 Yu-Poth S, Zhao G, Etherton T, Naglak M, Jonnalagadda S, Kris-Etherton PM. Effects of the National Cholesterol Education Program's Step I and Step II dietary intervention programs on cardiovascular disease risk factors: a metaanalysis. Am. J. Clin. Nutr. 1999; 69: 632-46.

2 Obesity: preventing and managing the global epidemic. Report of a WHO Consultation on Obesity, Geneva 3-5 June 1997. Geneva: WHO Publications, 1997.

3 Astrup A. The American paradox: the role of energy-dense fat-reduced food in the increasing prevalence of obesity. Curr. Opin. Clin. Nutr. Metab. Care 1998; 1: 573-7.
4 Prentice AM, Jebb SA. Obesity in Britain: gluttony or sloth? Br. Med.J. 1995; 311: 437-9.

5 Flatt JP, Ravussin E, Acheson KJ, Jequier E. Effects of dietary fat on postprandial substrate oxidation and on carbohydrate and fat balances. J. Clin. Invest. 1985; 76: 1019-24.

6 Astrup A, Flatt JP. Metabolic determinants of body weight regulation. In: Bouchard C, Bray GA, eds. Regulation of Body Weight, Biological and Behavioral Mechanisms. Dahlem Workshop. Report LS 57. Chichester: John Wiley \& Sons, 1996, 193-210.

7 Zhang $\mathrm{Y}$, Proenca R, Maffei M, Barone M, Leopold L, Friedman JM. Positional cloning of the obese mouse gene and its human homologue. Nature 1994; 372: 425-32.

8 Astrup A, Raben A, Buemann B, Toubro S. Fat metabolism in the predisposition to obesity. Ann. NY Acad. Sci. 1997; 827: 427-33.

9 Blundell JE, MacDiarmid JI. Passive overconsumption. Fat intake and short-term energy balance. Ann. NY Acad. Sci. 1997; 827: 392-407.

10 Stubbs RJ, Harbron CG, Murgatroyd PR, Prentice AM. Covert manipulation of dietary fat and energy density: effect on substrate flux and food intake in men eating ad libitum. $\mathrm{Am}$. J. Clin. Nutr. 1995; 62: 316-29.

11 Stubbs RJ, Ritz P, Coward WA, Prentice AM. Covert manipulation of the dietary fat to carbohydrate ratio and energy density: effect on food intake and energy balance in free-living men, feeding ad libitum. Am. J. Clin. Nutr. 1995; 62: $330-7$.

12 Tordoff MG, Reed DR. Sham-feeding sucrose or corn oil stimulates food intake in rats. Appetite 1991; 17: 97-103.

13 van Stratum P, Lussenberg RN, van Wezel LA, Vergoesen AJ, Cremer HD. The effect of dietary carohydrate: fat ratio on energy intake by adult women. Am. J. Clin. Nutr. 1978; 31: 206-12.

14 Astrup A, Toubro S, Raben A, Skov R. The role of low fat diets and fat substitutes in body weight management: what have we learned from clinical studies? J. Am. Diet. Assoc. 1997; 97: S82-7.

15 Astrup A. Dietary composition, substrate balances and body fat in subjects with a predisposition to obesity. Int. J. Obes 1993; 17(suppl. 3): S32-6.

16 Kant AK, Graubard BI, Schatzkin A, Ballard-Barbash R Proportion of energy intake from fat and subsequent weight change in the NAHNES 1 Epidemiologic Follow-up Study. Am. J. Clin. Nutr. 1995; 61: 11-7.

17 Prentice AM, Black AE, Coward WA, Davies Hl, Goldberg GR, Murgatroyd PR et al. High levels of energy expenditure in obese women. Br. Med. J. 1986; 292: 983-92.

18 Heitmann BE, Lissner L. Dietary underreporting by obese individuals-is it specific or non-specific? Br. Med.J. 1995; 311: 986-9.

19 Radimer KL, Harvey PWJ. Comparison of self-report of reduced fat and salt foods with sales and supply data. Eur.J. Clin. Nutr. 1998; 52: 380-2.

20 Paeratakul S, Popkin BM, Keyou G, Adeir LS, Stevens J. Changes in diet and physical activity affect the body mass index of Chinese adults. Int. J. Obes. 1998; 22: 424-31.

21 Bray GA, Popkin BM. Dietary fat intake does affect obesity. Am. J. Clin. Nutr. 1998; 68: 1157-73.

22 Astrup A, Ryan L, Grunwald G, Storgaard M, Saris W, Hill JO. Ad libitum low-fat diets and body fatness: a meta-analysis of intervention studies. J. A. M. A. (submitted).

23 Willet WC. Is dietary fat a major determinant of body fat? Am. J. Clin. Nutr. 1998; 67: 556s-62s.

24 Katan MB, Grundy SM, Willett WC. Beyond low-fat diets N. Engl.J. Med. 1997; 337: 563-6.

25 Rolls BJ, Rowe EA, Turner RC. Persistent obesity in rats following a period of consumption of a mixed, high energy diet. J. Physiol. (London) 1980; 298: 415-27.

26 Hill JO, Lin D, Yakubu F, Peters JC. Development of dietary 
obesity in rats: influence of amount and composition of dietary fat. Int. J. Obes. 1992; 16: 321-33.

27 Nagle DL, McGrail SH, Vitale J, Woolf EA, Dussault BJ, DiRocco L, Holmgren L, Montegno J, Bork P, Hugzar D, Fairchild-Huntress V, Ge P, Keiltz J, Ebeling C, Balbini L, Gilchrist J, Burn P, Carlson GA, Moore KJ. The mahogany protein is a receptor involved in suppression of obesity. Nature 1999; 398: 148-52.

28 Heitmann BL, Lissner L, Sørensen TIA, Bengtsson C. Dietary fat intake and weight gain in women genetically predisposed to obesity. Am. J. Clin. Nutr. 1995; 61: 1213-7.

29 Toubro S, Sørensen TIA, Hindsberger C, Christensen NJ, Astrup A. Twenty-four-hour respiratory quotient: the role of diet and familial resemblance. J. Clin. Endocrinol. Metab. 1998; 83: 2758-64.
30 Ranneries C, Bülow J, Buemann B, Christensen NJ, Madsen J, Astrup A. Fat metabolism in formerly obese women: effect of exercise on substrate oxidation and adipose tissue lipolysis. Am. J. Physiol. (Endocrinol. Metab.) 1998; 274: 155-61.

31 Raben A, Mygind E, Astrup A. Lower activity of oxidative key enzymes and smaller fiber areas in skeletal muscle of postobese women. Am. J. Physiol. 1998; 275: E487-94.

32 Heini AF, Weinsier RL. Divergent trends in obesity and fat intake patterns: the American paradox. Am. J. Med. 1997; 102: 259-64

33 Astrup A, Lundsgaard C, Stock MJ. Is obesity contagious? Int. J. Obes. 1998; 22: 375-6. 